\title{
Construction of the Fingerprints of Ginseng Stem and Leaf Saponin Reference Substances and Spiked Plasma Sample by LC-ESI/MS and Its Application to Analyzing the Compounds Absorbed into Blood after Oral Administration of Ginseng Stem and Leaf Saponin in Rat
}

\author{
Bei Yan, Guangji Wang, ${ }^{*}$ Jiye A, Lin XIE, Haiping Hao, Yan Liang, Jianguo Sun, Xiaoyu Li, and \\ Yuanting ZHENG \\ Key Laboratory of Drug Metabolism and Pharmacokinetics, China Pharmaceutical University; NO. 24, Tong Jia Xiang \\ Street, Nanjing 210009, P. R. China. $\quad$ Received May 1, 2007; accepted May 30, 2007
}

\begin{abstract}
Develop a simple and reliable assay method to detect ginseng stem and leaf saponins (GSLS) in methanol and rat plasma by liquid chromatography-electrospray ionization mass spectrometry in scan mode, and construct the fingerprints of GSLS reference substances and plasma samples. In order to screen the active constituents of GSLS, analysis and comparison were carried out between the LC/ESI-MS profiles of blank rat plasma and rat plasma samples obtained after oral administration of GSLS. Thirty-one compounds were detected and 10 of them were identified in the fingerprints of reference substances and spiked plasma sample. Fur-

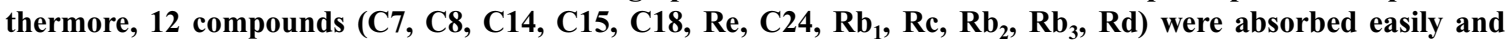
some new compounds were generated after oral administration of GSLS, which might be the metabolites of GSLS. These absorbed components and new compounds may be the main bioactive components of GSLS.
\end{abstract}

Key words ginseng saponin; fingerprint; rat plasma; LC-ESI/MS

Ginseng, the root of Panax ginseng C. A. MeYer (Araliaceae), has been used worldwide as an herbal medicine. Studies have proven that pharmacological properties of ginseng are generally attributed to triterpene glycosides, known as ginsenosides. They are the derivatives of either protopanaxadiol or protopanaxatriol group depending on different aglycones (Fig. 1). Untill now, more than 40 individual ginsenosides have been identified. Ginsenosides can postpone aging and delay the occurrence of exercise-induced fatigue. It has been reported that ginseng stem and leaf saponins (GSLS) had the similar pharmacological effects to ginseng

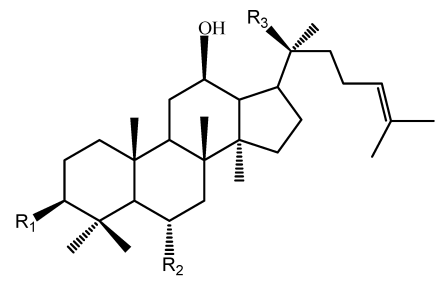

\begin{tabular}{|c|c|c|c|c|}
\hline Compound & $\mathrm{R}_{1}$ & $\mathrm{R}_{2}$ & $\mathrm{R}_{3}$ & $\begin{array}{c}\text { Molecular } \\
\text { weight }\end{array}$ \\
\hline \multicolumn{5}{|l|}{ Protopanaxadiol-type } \\
\hline Ginsenoside $-R b_{1}$ & $-\mathrm{O}-\mathrm{Glc}^{2}-{ }^{1} \mathrm{Glc}$ & $-\mathrm{H}$ & $-\mathrm{O}-\mathrm{Glc}^{6}-{ }^{1} \mathrm{Glc}$ & 1109 \\
\hline $\mathrm{Rb}_{2}$ & $-\mathrm{O}-\mathrm{Glc}^{2}-{ }^{1} \mathrm{Glc}$ & $-\mathrm{H}$ & -O-Glc ${ }^{6}-{ }^{1}$ Arap & 1079 \\
\hline $\mathrm{Rb}_{3}$ & $-\mathrm{O}-\mathrm{Glc}^{2}-{ }^{1} \mathrm{Glc}$ & $-\mathrm{H}$ & $-O-G c^{6}-{ }^{1} X y l$ & 1095 \\
\hline $\mathrm{Rc}$ & $-\mathrm{O}-\mathrm{Glc}^{2}-{ }^{1} \mathrm{Glc}$ & $-\mathrm{H}$ & -O-Glc ${ }^{6}{ }^{-1}$ Araf & 1079 \\
\hline $\mathrm{Rd}$ & $-\mathrm{O}-\mathrm{Glc}^{2}-{ }^{1} \mathrm{Glc}$ & $-\mathrm{H}$ & -O-Glc & 947 \\
\hline $\mathrm{Rg}_{3}$ & $-\mathrm{O}-\mathrm{Glc}^{2}-{ }^{1} \mathrm{Glc}$ & $-\mathrm{H}$ & $-\mathrm{OH}$ & 784 \\
\hline 20(S)-protopanaxadiol & $-\mathrm{OH}$ & $-\mathrm{H}$ & $-\mathrm{OH}$ & 460 \\
\hline \multicolumn{5}{|l|}{ Protopanaxatriol-type } \\
\hline Ginsenoside -Re & $-\mathrm{OH}$ & $-\mathrm{O}-\mathrm{Glc}^{2}-{ }^{1} \mathrm{Rha}$ & -O-Glc & 946 \\
\hline $\mathrm{Rg}_{1}$ & $-\mathrm{OH}$ & -O-Glc & -O-Glc & 800 \\
\hline $\mathrm{Rg}_{2}$ & $-\mathrm{OH}$ & $-\mathrm{O}-\mathrm{Glc}^{2}-{ }^{1} \mathrm{Rha}$ & $-\mathrm{OH}$ & 784 \\
\hline $\mathrm{Rf}$ & $-\mathrm{OH}$ & $-\mathrm{O}-\mathrm{Glc}^{2}-{ }^{1} \mathrm{Glc}$ & $-\mathrm{OH}$ & 800 \\
\hline $\mathrm{Rh}_{1}$ & $-\mathrm{OH}$ & -O-Glc & $-\mathrm{OH}$ & 638 \\
\hline Notoginsenoside- $\mathrm{R}_{1}$ & $-\mathrm{OH}$ & $-\mathrm{Glc}^{2}{ }^{-1} \mathrm{Xyl}$ & -Glc & 933 \\
\hline 20(S)-protopanaxatriol & $-\mathrm{OH}$ & $-\mathrm{OH}$ & $-\mathrm{OH}$ & 476 \\
\hline
\end{tabular}

Fig. 1. Chemical Structures and Molecular Weight Values of GSLS root saponins. ${ }^{1-3)}$

The studies of the pharmacological actions of GSLS have achieved great progress. Questions that what components can absorb into the circulatory system and which of them have pharmacological actions are still a riddle. Because of the complexity of GSLS and low sensitivities of detectors, several pharmacokinetics studies on GSLS reported only focused on single or several saponins, thus causing ignore the synergistic reactions among multicomponents of GSLS. In order to illuminate the active components of GSLS, pharmacokinetics and pharmacodynamics studies should be integrated well. And that which components can be absorbed into blood after oral administration of GSLS should be elucidated.

In 1988, a Japanese researcher named Tashiro Shin-ichi proposed the concept of "serum pharmacochemistry" and "serum pharmacology". Serum pharmacochemistry is such a branch of science that analyzes and identifies the main components absorbed in the circulation system after administration of Traditional Chinese Medicine (TCM), that screens and monitors active components to avoid screening active components blindly, that combining with pharmacokinetics and pharmacodynamics, illuminates the regularities of the actions and metabolism of TCM.

In this study, as serum pharmacochemistry a guidance, a new LC-ESI/MS method in scan mode to detect and identify GSLS in rat plasma simultaneously and globally was developed. Our work mainly included preliminary construction of the fingerprints of GSLS reference substances and plasma after oral administration in rats and qualitative analysis of the main components absorbed in rat circulatory system.

\section{MATERIALS AND METHODS}

Chemicals, Reagents and Animals GSLS reference substances (purity $>90 \%$ ) were supplied by Norman 
Bethune University of Medical Sciences. Methanol and acetonitrile were of HPLC grade from Merck (Darmstadt, Germany). Water was purified by a Milli-Q system (Millipore, Bedford, MA, U.S.A.). Other reagents were highest purity commercially available.

Sprague-Dawley rats, weighing varying from 180 to $220 \mathrm{~g}$, were obtained from Shanghai SATPTBE-PK Lab Animal Co., Ltd. (Shanghai, China). The study was conducted in accordance with the Animal Ethics Committee of China Pharmaceutical University. The animals were cared in accordance with the regulations of the Animal Committee under controlled conditions $\left(20 \pm 2{ }^{\circ} \mathrm{C}\right.$, RH $50 \pm 20 \%$, a natural lightdark cycle and $10-15$ air changes per hour).

Apparatus A Shimadzu 2010A LC-MS system with ESI interface, hyphened with a Shimadzu LC-10AD HPLC system equipped with an autosampler (SIL-HTc), two Shimadzu LC-10ADvp pumps, a Shimadzu CTO-10Avp column oven and a Shimadzu DGU-14AM online degasser was used for HPLC/MS analysis. A Q-array-octapole-quadrupole mass analyzer was used as the detector. And Shimadzu LCMSsolution Workstation software (Ver 2.02) for the data processing were utilized to perform the analytical procedures. SORVALL Biofuge Stratos (Germany) was used to centrifuge. SPD2010-230 SpeedVac Concentrator (Thermo Savant, U.S.A.) was used to evaporate the extracted organic solvents to dryness.

Instrumentation and Operating Conditions Separation was carried out using a shim-pack VP-ODS column $(2.0$ $\mathrm{mm} \times 250 \mathrm{~mm}, 4.6 \mu \mathrm{m}$, Shimadzu, Japan) with a C18 guard column (Security Guard, Phenomenex, U.S.A.) at $40{ }^{\circ} \mathrm{C}$. The mobile phase, at the flow rate of $0.2 \mathrm{ml} / \mathrm{min}$, was made up of acetonitrile (solvent B) and water containing $1 \mathrm{mmol} / \mathrm{l}$ of ammonium chloride (solvent A) under an elution program (Table 1). The MS acquisition was performed in scan mode of negative ions. The scan range was divided into two segments: $m / z \quad 450-850$ and $m / z \quad 850-1250$. The specific ions of GSLS were detected as their chloride adduct ions, $[\mathrm{M}+\mathrm{Cl}]^{-}$mainly. GSLS were identified by comparing their $[\mathrm{M}+\mathrm{Cl}]^{-}$ions and their retention times with those of authentic standards available in our lab. The optimized MS operating conditions were selected as follows: CDL (curved desolvation line) temperature: $250{ }^{\circ} \mathrm{C}$; the block temperature: $200{ }^{\circ} \mathrm{C}$; the probe temperature: $250{ }^{\circ} \mathrm{C}$; detector gain: $1.65 \mathrm{kV}$; probe voltage: $-3.5 \mathrm{kV}$; CDL voltage: $15 \mathrm{~V}$; Q-array $\mathrm{DC}$ (direct current) voltage: $0 \mathrm{~V}$; RF (radiation frequency) voltage: $150 \mathrm{~V}$. Nitrogen (Gas Supplier Center of Nanjing University, China) was served as nebulizer gas (flow rate: 1.5 $1 \mathrm{~min}^{-1}$ ) and curtain gas (pressure: $1 \mathrm{MPa}$ ).

Preparation of Stock Solution and GSLS-Spiked Plasma Sample The stock standard solution containing 0.1 $\mathrm{mg} / \mathrm{ml} \mathrm{GSLS}$ was prepared in methanol and stored at $4{ }^{\circ} \mathrm{C}$.

Add appropriate diluted stock solution of GSLS to Eppendorf tube, and evaporate to dryness, then add appropriate amounts of blank rat plasma to yield GSLS-spiked plasma sample in $25 \mu \mathrm{g} / \mathrm{ml}$.

Extraction Procedures $0.5 \mathrm{ml}$ of plasma was subjected to solid-phase extraction using OASIS HLB cartridges from Waters corporation (Milford, Mass U.S.A.) after equilibrating with $1 \mathrm{ml}$ of methanol and $1 \mathrm{ml}$ of water. Under gentle vacuum the solvent was passed through the cartridge, which was then washed three times with water and dried thereafter. Fi-
Table 1. Solvent Composition of the Linear Gradient of the LC-ESI/MS System

\begin{tabular}{ccc}
\hline \hline Time $(\mathrm{min})$ & $\begin{array}{c}\text { Acetonitrile } \\
(\%)\end{array}$ & $\begin{array}{c}\text { Water }(1 \mathrm{mmol} / \mathrm{l} \\
\text { ammonium chloride })(\%)\end{array}$ \\
\hline 0.03 & 15 & 85 \\
7.00 & 30 & 70 \\
35.00 & 50 & 50 \\
40.00 & 80 & 20 \\
43.00 & 80 & 20 \\
46.00 & 15 & 85 \\
55.00 & 15 & 85 \\
\hline
\end{tabular}

nally, the analyte was eluted with $1 \mathrm{ml}$ of $70 \%$ methanol, followed by $1 \mathrm{ml}$ of methanol. These eluents were mixed and 10 $\mu \mathrm{l}$ was subjected to mass spectrometric analysis.

Animal Study Design and Procedure Male rats with the average weight of $200 \mathrm{~g}$ were fasted for $12 \mathrm{~h}$ before dosing and $4 \mathrm{~h}$ afterward, while water was taken freely. The dosage of GSLS was $500 \mathrm{mg} / \mathrm{kg}$ with the volume of 0.5 $\mathrm{ml} / 100 \mathrm{~g}$, administered orally. Blood samples (about $300 \mu \mathrm{l}$ ) were collected in heparinized $1.5 \mathrm{ml}$-polythene tubes immediately before dosing and at 1, 4, 8, 12, 24, 36 h after dosing, then centrifuged at $4000 \mathrm{rpm}$ for $5 \mathrm{~min}$. The plasma obtained was stored at $-20^{\circ} \mathrm{C}$ until analysis. Plasma sample is often used in pharmacokinetics and more plasma than serum can be obtained from blood with equal volume, so in this experiment, plasma was used instead of serum.

\section{RESULTS AND DISCUSSION}

Development and Optimization of Determination Method The bioavailability of perorally administered GSLS is extremely low, and their blood concentration are always too low to be detected. Mass spectrometry is a sensitive detector, therefore, HPLC hyphened mass spectrometry was adopted to determine GSLS in rat plasma. The preliminary experiment found that the signal intensity of GSLS is stronger in LC/ESI-MS than that in LC/APCI-MS, because LC/APCI-MS is more suitable to detect compounds with low polarity, while GSLS with several glycons have higher polarity.

The responses of GSLS to ESI were evaluated by the mass spectra scanned from $\mathrm{m} / \mathrm{z} 450$ to 1250 which could include all the GSLS and their metabolites in both negative and positive ionization modes. Studies showed that it could be detected more peaks in negative mode than in positive mode. Negative mode mainly yielded signals of $[\mathrm{M}+\mathrm{Cl}]^{-}$of GSLS. Due to the high noise occurred in the negative scan mode, the scan range of the chloride adduct ion, $[\mathrm{M}+\mathrm{Cl}]^{-}$of GSLS, was finally divided into two segments: $m / z \quad 450-850$ and $m / z 850-1250$ to increase the responses.

Two kinds of available chromatographic columns in different length, diameter and brand were utilized to analyze. It was found that using Shimadzu $\mathrm{C}_{18}$ column $(250 \mathrm{~mm} \times 2.0$ $\mathrm{mm}, 5 \mu \mathrm{m}$, Japan) could separate more peaks and gain more information than using Kromasil $\mathrm{C}_{18}$ column $(150 \mathrm{~mm} \times 2.1$ $\mathrm{mm}, 3.5 \mu \mathrm{m}$, U.S.A.), for short column had lower column efficiency than long column, especially when the analysis time was long.

Tests proved obviously that acetonitrile had much better 
eluting power than methanol, so acetonitrile was selected as the organic phase. Considering LC-ESI/MS was set in negative ionization mode, ammonium acetate and ammonium chloride, prone to increase the signal intensity of negative ions, were chosen to add into water in different concentrations as the aqueous phase. Tests demonstrated that water containing ammonium chloride could enhance the response of GSLS and detecting stability. Therefore, mobile phase consisted of acetonitrile and water $(1 \mathrm{mmol} / 1$ ammonium chloride) was employed. Abundant information, strong response, suitable retention time and good stability were also obtained on the condition of gradient elution which was a key to construct fingerprints. The developed method was successfully employed for detection of GSLS in methanol and rat plasma globally.

Selection of the Extraction Method In serum pharmacochemistry research, it is expected to detect the maximal components in serum, so it must select the suitable extraction method to decrease the loss of components in rat plasma during extraction. Moreover, GSLS had the similar structures, but their polarities were different due to the numbers of sugar moieties binding to aglycone, and so do their metabolites. Therefore, direct deproteinization and solid phase extraction (SPE) may be the main sample preparation for GSLS in plasma or serum.

Methanol direct deproteinization had been investigated, and it was found that most of GSLS cannot be detected. Fortunately, our laboratory has previously reported SPE method for extracting GSLS. ${ }^{5-7)}$ Gradient elution in SPE could be applied to solve the problems about different polarities. Furthermore, the extracted sample would be clean enough not to block the detector, resulting in prolonged use of mass spectrometry detector accordingly. 10\%, 20\%, 30\%, 40\%, 50\%, $60 \%, 70 \%, 80 \%, 90 \%$ and $100 \%$ methanol as eluant were tested. Finally, using $70 \%$ and $90 \%$ methanol in order could elute GSLS with different polarity and the maximal chromatographic peaks could be detected.

LC/ESI-MS Fingerprinsts of GSLS Reference Substances Using liquid chromatography-electrospray ionization mass spectrometry in scan mode of negative ions in the range of $\mathrm{m} / \mathrm{z} 450-850$ and $\mathrm{m} / \mathrm{z} 850-1250$ and using solvent gradient elution system comprised by acetonitrile and purified water containing $1 \mathrm{mmol} / \mathrm{l}$ ammonium chloride, the LC/ESI-MS fingerprints of GSLS reference substances were obtained (see Fig. 2). And only when the component's intensity exceeds 15000 can I consider it as a peak.

As summarized in Table 2, in the range of $m / z 450-850$, 16 peaks labelled $1-16$ include 21 components $(\mathrm{C} 1-\mathrm{C} 21)$. In the TIC chromatogram, chromatographic peaks of more than one main component were often overlapped and formed one peak, thus, in fact, peak 3 was formed by two overlapped peaks, namely components labelled $\mathrm{C} 3$ and $\mathrm{C} 4$. Peak 8 was formed by two overlapped peaks, namely components labeled $\mathrm{C} 9$ and $\mathrm{C} 10$. Peak 10 were formed by three overlapped peaks, namely components labelled C12, C13 and C14. Peak 13 was formed by two overlapped peaks which emerged from 2 components labelled $\mathrm{C} 17$ and $\mathrm{C} 18$. In the range of $\mathrm{m} / \mathrm{z}$ $850-1250,10$ peaks labelled $1-10$ include 10 components (C22-C31). Comparison of the $\mathrm{m} / z$, retention time of the sample with that of ginsenosides standards available in our laboratory, it could be identified that $\mathrm{C} 3, \mathrm{C} 17, \mathrm{C} 21 \mathrm{C} 22$,
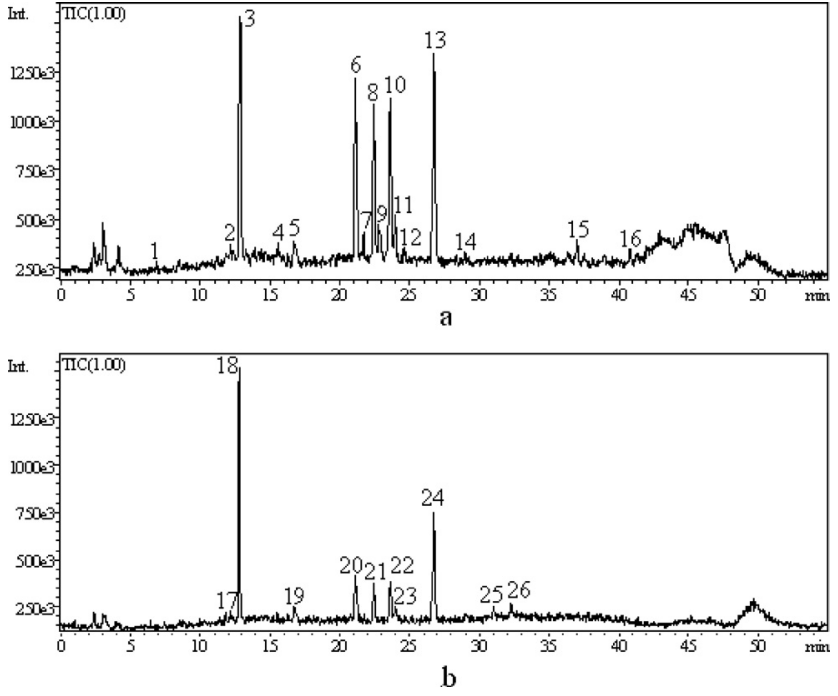

Fig. 2. LC-ESI/MS Fingerprints of GSLS Reference Substance

Table 2. The Main Peaks and Components Detected in the Fingerprints of GSLS Reference Substances.

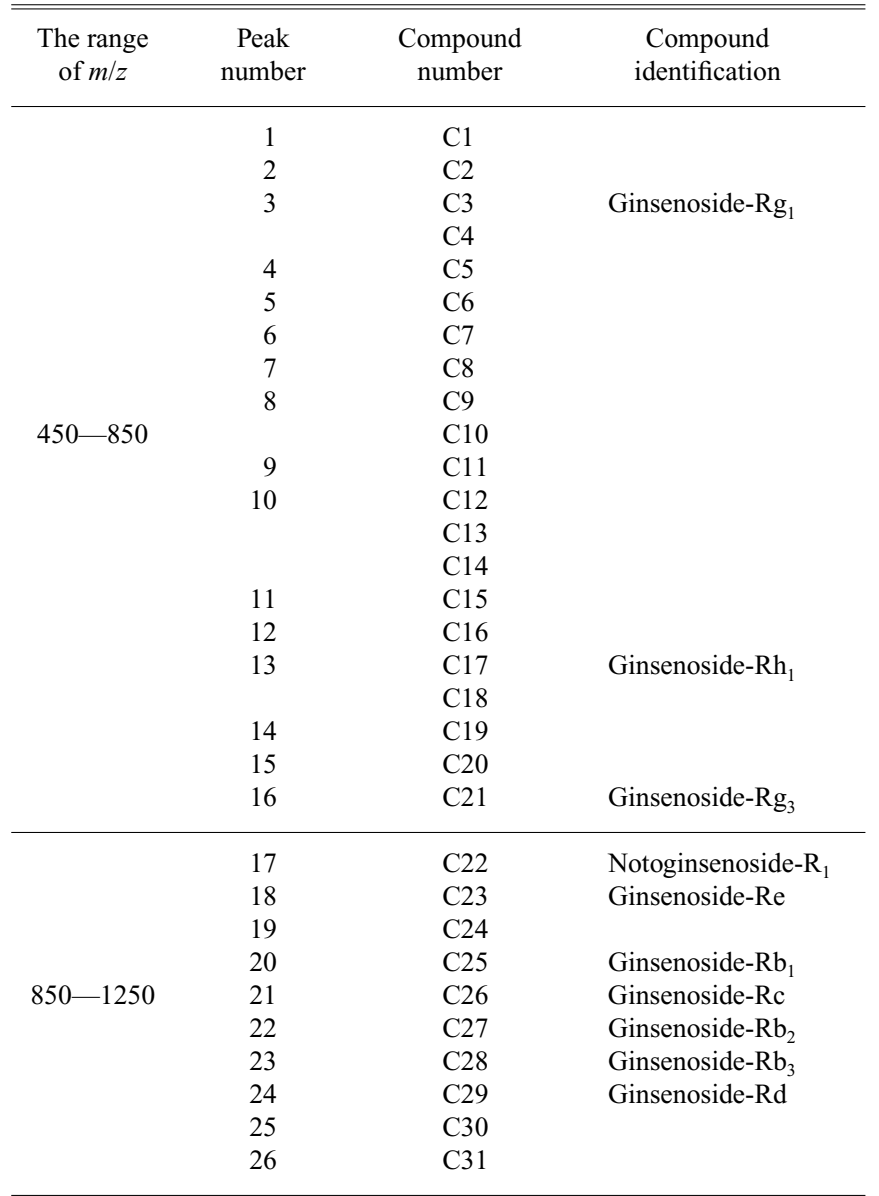

C23, C25, C26, C27, C28 and C29 were ginsenoside- $\mathrm{Rg}_{1}$, ginsenoside- $\mathrm{Rh}_{1}$, ginsenoside- $\mathrm{Rg}_{3}$, notoginsenoside- $\mathrm{R}_{1}$, ginsenoside- $R e$, ginsenoside- $R b_{1}$, ginsenoside- $R c$, ginsenoside$R b_{2}$, ginsenoside- $R b_{3}$, and ginsenoside- $R d$, respectively.

Methodology Validation LC-MS fingerprint determination is usually different from general assaying method. The authentication and identification of a drug and its products 
can be accurately performed using the chromatographic fingerprints, even if batches or concentrations varied among samples. ${ }^{8)}$ Considering these characteristics of fingerprints, Peak 6 (C7), Peak 11(C15), Peak 18 (Re) and Peak 24 (Rd) with high signal intensity and without overlapped peaks were used to evaluate the assay method.

The injection precision was determined by replicating injections of the GSLS reference substances and extracted GSLS spiked-plasma sample six times in $1 \mathrm{~d}$. In reference substances, the relative standard deviations of ratios of $\mathrm{C} 7$, $\mathrm{C} 15, \mathrm{Re}$ and $\mathrm{Rd}$ peak areas were lower than $6.86 \%, 7.77 \%$, $8.23 \%$ and $7.73 \%$, respectively. In extracted plasma sample, the relative standard deviations of ratios of $\mathrm{C} 7, \mathrm{C} 15, \mathrm{Re}$ and $\mathrm{Rd}$ peak areas were lower than $7.95 \%, 7.82 \%, 6.67 \%$ and $6.95 \%$, respectively.

The repeatability was assessed by analyzing six independently prepared GSLS reference substances and extracted GSLS spiked-plasma sample. In reference substances, the RSDs of $\mathrm{C} 7, \mathrm{C} 15, \mathrm{Re}$ and $\mathrm{Rd}$ peak areas were lower than $9.99 \%, 12.02 \%, 9.45 \%$ and $10.37 \%$, respectively. In extracted GSLS spiked-plasma sample, the RSDs of C7, C15, $\mathrm{Re}$ and $\mathrm{Rd}$ peak areas were lower than $14.23 \%, 13.01 \%$, $13.98 \%$ and $14.51 \%$, respectively.

The sample stability test was determined with one GSLS reference substance sample during $3 \mathrm{~d}$ at room temperature and one GSLS spiked-plasma sample after storage at room temperature for $6 \mathrm{~h}$, after three freeze $\left(-20^{\circ} \mathrm{C}\right)$ and thaw (room temperature) cycles. In reference substances, the RSDs of $\mathrm{C} 7, \mathrm{C} 15, \mathrm{Re}$ and $\mathrm{Rd}$ peak areas were lower than $9.36 \%, 11.33 \%, 10.99 \%, 12.32 \%$, respectively. In extracted GSLS spiked-plasma sample, the RSDs of C7, C15, Re and $\mathrm{Rd}$ peak areas were lower than $13.87 \%, 14.12 \%, 11.99 \%$, $13.01 \%$, respectively. The results indicated that the reference substance and plasma sample remained stable under the condition mentioned above.

The extraction recovery was evaluated by comparing the peak areas of $\mathrm{C} 7, \mathrm{C} 15, \mathrm{Re}$ and $\mathrm{Rd}$ obtained from six independently GSLS spiked-plasma sample which were subjected to the extraction procedure with those obtained from the same nominal concentrations $(5.0 \mu \mathrm{g} / \mathrm{ml})$ of reference substance solution mixed with blank plasma extracts. The recoveries of $\mathrm{C} 7, \mathrm{C} 15, \mathrm{Re}$ and $\mathrm{Rd}$ in the concentration range of 5.0 $\mu \mathrm{g} / \mathrm{ml}$ were all $>80 \%$, with $\mathrm{RSD} \%$ of $<15 \%$. This suggested that recoveries of $\mathrm{C} 7, \mathrm{C} 15, \mathrm{Re}$ and $\mathrm{Rd}$ were consistent, precise and reproducible during the validation experiments.

All results from injection precision, repeatability, stability and extraction recovery test indicate that this method was adequate, valid and applicable.

LC/ESI-MS Fingerprints of GSLS-Spiked Plasma SPE was used to extract GSLS from plasma and studies have proved it had the higher extraction efficiency and less loss than direct deproteinization. Figure 3 showed the LC/ESIMS fingerprints of GSLS-spiked plasma $(25 \mu \mathrm{g} / \mathrm{ml})$ after extraction. The peaks detected in the fingerprints of GSLS reference substances were all detected in the fingerprints of GSLS-spiked plasma.

LC/ESI-MS Fingerprinsts of Plasma Samples after Oral Administration of GSLS to Rats Rat plasma samples obtained in different time points after oral administration of GSLS were analyzed. Fingerprints of plasma samples in different time points were diverse (Take one rat for exam-
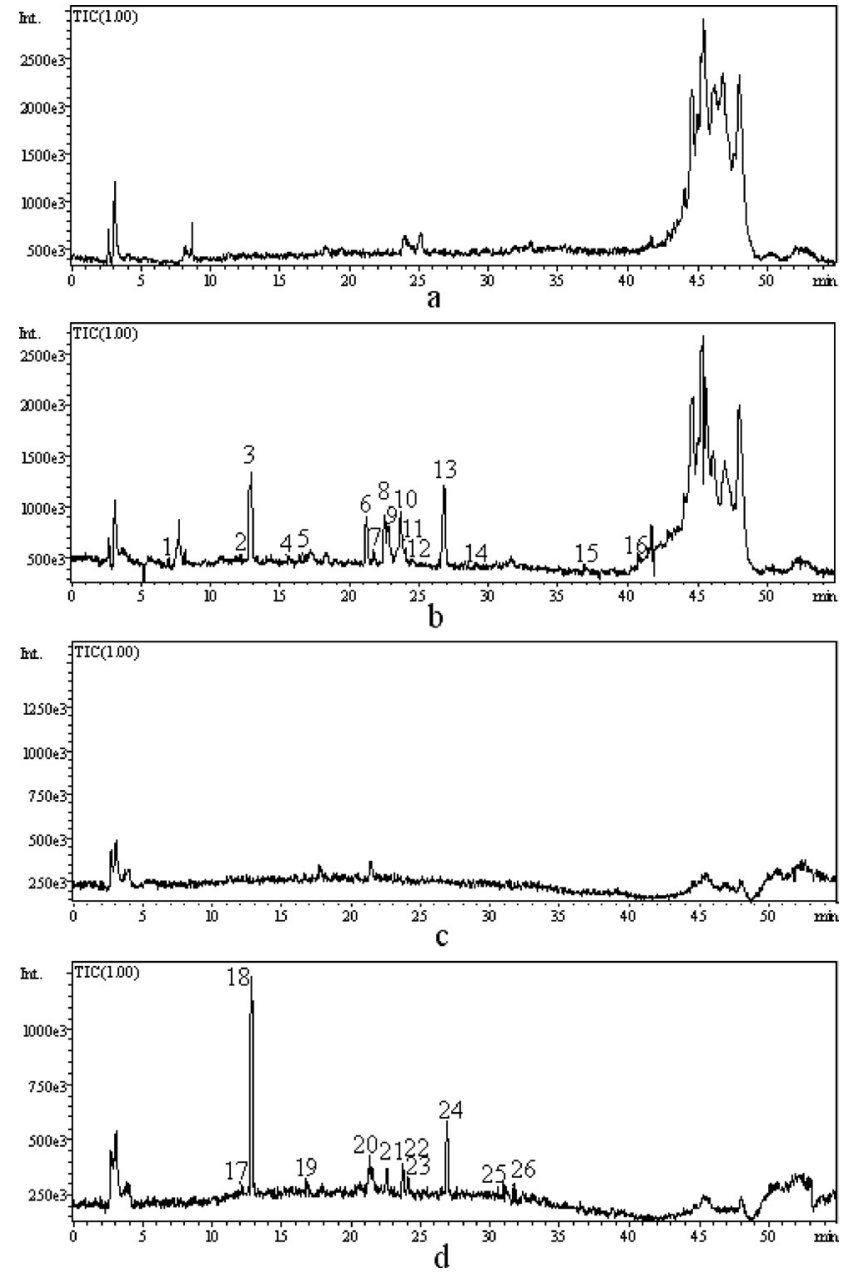

Fig. 3. LC-ESI/MS Fingerprints of GSLS-Spiked Plasma

ple in Fig. 4). Comparing the LC/ESI-MS profiles of the plasma samples with those of blank rat plasma and GSLS reference substances, it was found that some of the components in GSLS reference substances could be found in the plasma samples in different time, which meant different components were absorbed into rat blood in different time points, and that the kinds and the amounts of the absorbed components changed dynamically, yet the changing regularity would be elucidated clearly in further studies. It was also found that two new peaks at $8.1 \mathrm{~min}$ and at $17.5 \mathrm{~min}$ were appeared in the fingerprints of plasma samples in the range of $m / z 850-1250$. The new peaks may be formed by the metabolites of GSLS or the endogenous stress metabolites caused by GSLS, and their structures should be identified in further studies.

The study showed that individual variation existed in the LC/MS fingerprints of plasma samples, especially those components in low content, but the components with high content and more easily absorbed into rat blood may affect bioactivities mainly, so these components were the focus of our research. It could be detected 5, 5, 7, 6, 5 and 5 mainly common components in the range of $m / z 450-850$ in $1 \mathrm{~h}, 4$ $\mathrm{h}, 8 \mathrm{~h}, 12 \mathrm{~h}, 24 \mathrm{~h}$ and $36 \mathrm{~h}$ in all rats, respectively, and 7, 7, 7, 7,5 and 5 mainly common components in the range of $\mathrm{m} / \mathrm{z}$ $850-1250$ in $1 \mathrm{~h}, 4 \mathrm{~h}, 8 \mathrm{~h}, 12 \mathrm{~h}, 24 \mathrm{~h}$ and $36 \mathrm{~h}$ in all rats, respectively (Table 3 ). In other word, 12, 12, 14, 13, 10 and 10 

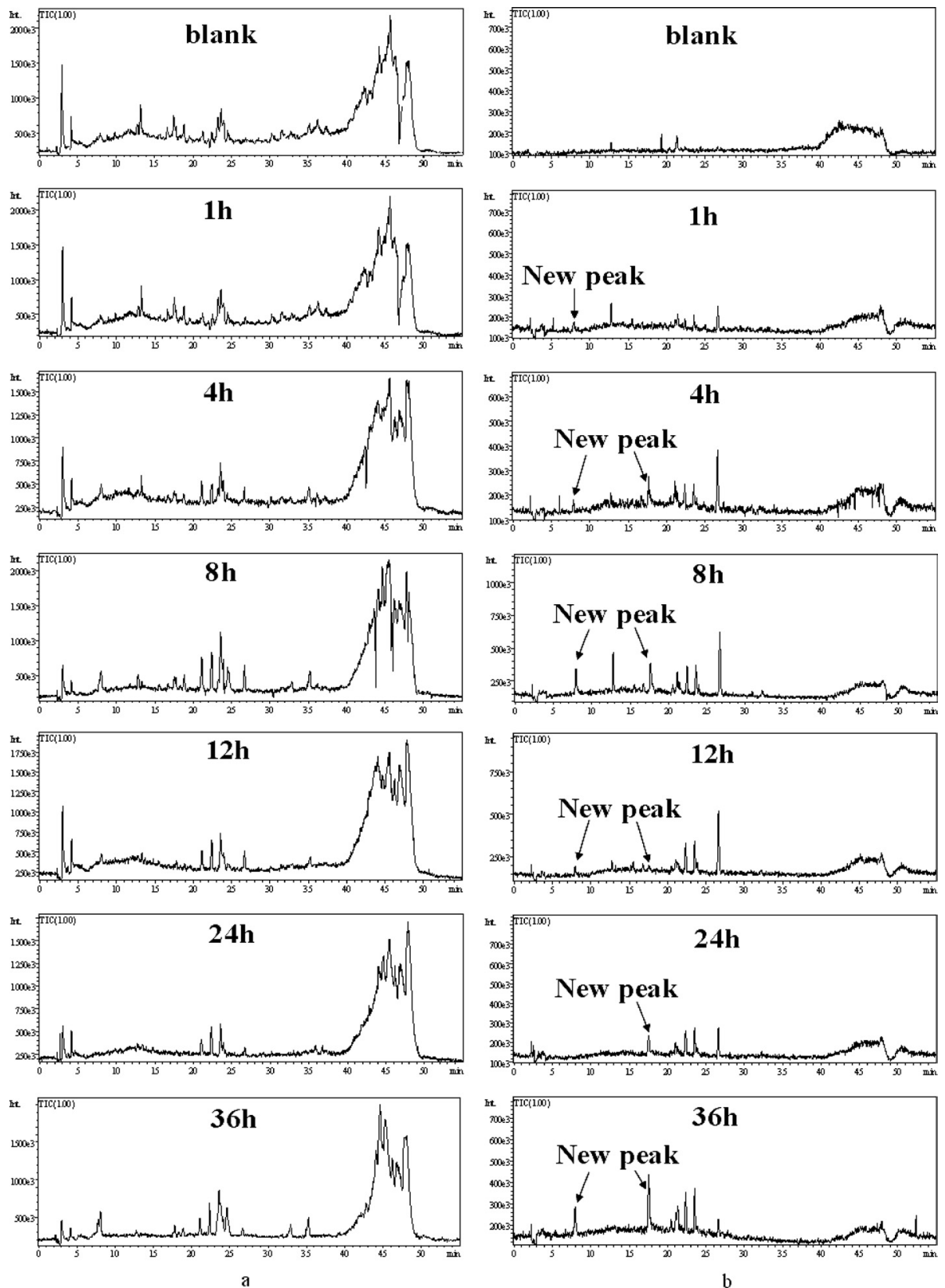

Fig. 4. LC-ESI/MS Fingerprints of Plasma Samples after Oral Administration of GSLS in Rats in $0 \mathrm{~h}, 1 \mathrm{~h}, 4 \mathrm{~h}, 8 \mathrm{~h}, 12 \mathrm{~h}, 24 \mathrm{~h}$ and $36 \mathrm{~h}$, Respectively

common components in all were absorbed in rat blood in $1 \mathrm{~h}$, $4 \mathrm{~h}, 8 \mathrm{~h}, 12 \mathrm{~h}, 24 \mathrm{~h}$ and $36 \mathrm{~h}$, respectively. Comparing the change of relative content of each component after and before absorbed in circulation system, it was discovered that C7, C9, C14, C15, C18, C23 (Re), C24, C25 (Rb 1$), C 26$ $(\mathrm{Rc}), \mathrm{C} 27\left(\mathrm{Rb}_{2}\right), \mathrm{C} 28\left(\mathrm{Rb}_{3}\right)$ and $\mathrm{C} 29(\mathrm{Rd})$ were absorbed more easily, eliminated more slowly and their relative content was higher after absorbed than that before absorbed, so these components and the metabolites may be the bioactive components.

\section{CONCLUSION}

Develop a simple, reliable and stable assay method to construct GSLS reference substance fingerprints and plasma fingerprints, and qualitatively analyze GSLS in methanol and rat plasma by liquid chromatography-electrospray ionization mass spectrometry in scan mode. Thirty-one components could be detected in the fingerprints of GSLS reference substances and 10 of them could be identified. Analyze the main components absorbed in rat blood after oral administration ginseng stem and leaf saponins. It was found that only several components of those 31 components were absorbed in circulatory system in different time points-12, 12, 14, 13, 10 and 10 common components in all could be absorbed into rat blood in $1 \mathrm{~h}, 4 \mathrm{~h}, 8 \mathrm{~h}, 12 \mathrm{~h}, 24 \mathrm{~h}$ and $36 \mathrm{~h}$, respectively.

Because of the different physico-chemical properties of drugs, when they pass the gastrointestinal tract, their characteristics of absorption differ naturally. The higher absorption ratio a drug has, the higher bioactivity it may have. In this study, C7, C9, C14, C15, C18, C23 (Re), C24, C25 (Rb $)$, C26 (Rc), C27 $\left(\mathrm{Rb}_{2}\right), \mathrm{C} 28\left(\mathrm{Rb}_{3}\right)$ and $\mathrm{C} 29(\mathrm{Rd})$ may be the bioactive components. The data present in this paper may be the base for the pharmacokinetics and pharmacodynamics re- 
Table 3. Components Absorbed in Rat Circulation System after Oral Administration of GSLS to Rats

\begin{tabular}{|c|c|c|c|c|c|c|c|c|c|c|c|c|c|c|c|c|c|c|}
\hline \multirow{2}{*}{ Component No. } & \multicolumn{6}{|c|}{ Rat 1} & \multicolumn{6}{|c|}{ Rat 2} & \multicolumn{6}{|c|}{ Rat 3} \\
\hline & $1 \mathrm{~h}$ & $4 \mathrm{~h}$ & $8 \mathrm{~h}$ & $12 \mathrm{~h}$ & $24 \mathrm{~h}$ & $36 \mathrm{~h}$ & $1 \mathrm{~h}$ & $4 \mathrm{~h}$ & $8 \mathrm{~h}$ & $12 \mathrm{~h}$ & $24 \mathrm{~h}$ & $36 \mathrm{~h}$ & $1 \mathrm{~h}$ & $4 \mathrm{~h}$ & $8 \mathrm{~h}$ & $12 \mathrm{~h}$ & $24 \mathrm{~h}$ & $36 \mathrm{~h}$ \\
\hline \multicolumn{19}{|l|}{$\mathrm{C} 1$} \\
\hline \multicolumn{19}{|l|}{$\mathrm{C} 2$} \\
\hline $\mathrm{C} 3\left(\mathrm{Rg}_{1}\right)$ & $*$ & $\triangle$ & $*$ & $*$ & & & $*$ & & $*$ & $*$ & & & $*$ & & $*$ & $*$ & & \\
\hline $\mathrm{C} 4$ & & & & $\triangle$ & & & $\triangle$ & $\triangle$ & $\triangle$ & & & & $\triangle$ & & $\triangle$ & $\triangle$ & & \\
\hline $\mathrm{C} 5$ & * & $\triangle$ & $*$ & $\triangle$ & & & $*$ & $\triangle$ & $*$ & $\triangle$ & & & $*$ & & $*$ & & & \\
\hline C6 & $\triangle$ & $\triangle$ & & & & & $\triangle$ & $\triangle$ & $\triangle$ & $\triangle$ & & & & & $\triangle$ & & & \\
\hline $\mathrm{C} 7$ & $*$ & $*$ & $*$ & $*$ & $*$ & $*$ & $*$ & $*$ & $*$ & $*$ & * & $*$ & $*$ & $*$ & $*$ & * & $*$ & $*$ \\
\hline \multicolumn{19}{|l|}{$\mathrm{C} 8$} \\
\hline $\mathrm{C} 9$ & $*$ & $*$ & $*$ & $*$ & $*$ & $*$ & $*$ & $*$ & $*$ & $*$ & $*$ & $*$ & $*$ & $*$ & $*$ & $*$ & $*$ & * \\
\hline \multicolumn{19}{|l|}{$\mathrm{C} 10$} \\
\hline $\mathrm{C} 11$ & & & & $\triangle$ & & & & & & & & & & & & & & \\
\hline \multicolumn{19}{|l|}{$\mathrm{C} 12$} \\
\hline $\mathrm{C} 13$ & & & & $\triangle$ & & & & & & & & & & & & & & \\
\hline $\mathrm{C} 14$ & $\triangle$ & * & * & * & * & * & & * & $*$ & * & * & * & $\triangle$ & $*$ & * & * & * & * \\
\hline $\mathrm{C} 15$ & $\triangle$ & $*$ & $*$ & * & * & * & & $*$ & $*$ & * & * & * & $\triangle$ & $*$ & $*$ & * & * & * \\
\hline $\mathrm{C} 16$ & $\triangle$ & & & & & & & & & & & & & & & & & \\
\hline \multicolumn{19}{|l|}{$\mathrm{C} 17\left(\mathrm{Rh}_{1}\right)$} \\
\hline C18 & * & $*$ & $*$ & * & * & $*$ & $*$ & $*$ & $*$ & $*$ & $*$ & $*$ & $*$ & $*$ & $*$ & $*$ & $*$ & $*$ \\
\hline \multicolumn{19}{|l|}{ C19 } \\
\hline \multicolumn{19}{|l|}{$\mathrm{C} 20$} \\
\hline \multirow{2}{*}{\multicolumn{19}{|c|}{$\mathrm{C} 21\left(\mathrm{Rg}_{3}\right)$}} \\
\hline $\mathrm{C} 22$ & & & & & & & & & & & & & & & & & & \\
\hline \multicolumn{19}{|l|}{$\left(\right.$ Notoginsenoside- $\left.\mathrm{R}_{1}\right)$} \\
\hline $\mathrm{C} 23(\mathrm{Re})$ & * & $*$ & $*$ & $*$ & & & $*$ & $*$ & $*$ & $*$ & & & $*$ & $*$ & $*$ & $*$ & & \\
\hline $\mathrm{C} 24$ & $\triangle$ & $*$ & $*$ & * & & & $\triangle$ & $*$ & $*$ & * & & & $\triangle$ & $*$ & * & * & & \\
\hline $\mathrm{C} 25\left(\mathrm{Rb}_{1}\right)$ & $*$ & $*$ & $*$ & $*$ & $*$ & $*$ & $*$ & $*$ & $*$ & $*$ & $*$ & $*$ & $*$ & $*$ & $*$ & * & $*$ & $*$ \\
\hline $\mathrm{C} 26(\mathrm{Rc})$ & $\triangle$ & $*$ & $*$ & * & * & * & & $*$ & $*$ & * & * & * & $\triangle$ & $*$ & * & * & * & * \\
\hline $\mathrm{C} 27\left(\mathrm{Rb}_{2}\right)$ & $\triangle$ & $*$ & $*$ & * & * & * & & * & $*$ & * & $*$ & * & $\triangle$ & $*$ & * & * & * & * \\
\hline $\mathrm{C} 28\left(\mathrm{Rb}_{3}\right)$ & $\triangle$ & $*$ & $*$ & * & $*$ & $*$ & & $*$ & $*$ & * & $*$ & $*$ & $\triangle$ & $*$ & $*$ & * & $*$ & * \\
\hline $\mathrm{C} 29(\mathrm{Rd})$ & $*$ & $*$ & $*$ & * & $*$ & $*$ & $*$ & $*$ & $*$ & $*$ & $*$ & $*$ & $*$ & $*$ & $*$ & * & $*$ & $*$ \\
\hline $\mathrm{C} 30$ & $\triangle$ & & & & & & & & & & & & & & $\triangle$ & & & \\
\hline $\mathrm{C} 31$ & $\triangle$ & & $*$ & $\triangle$ & & & & & $*$ & & & & & & $*$ & & & \\
\hline
\end{tabular}

*, the common components absorbed in circulation system of all the rats; $\triangle$, the special components absorbed in circulation system of certain rats.

searches on ginseng stem and leaf saponins and for explaining and judging their diverse bioactivities.

Acknowledgements The project was supported by National Nature Science Fund (30572228) and Jiangsu Nature Science Fund (BK2004111).

\section{REFERENCES}

1) Takino Y., Odani T., Tanizawa H., Chem. Pharm. Bull., 30, 21962201 (1982).

2) Pei Y. Q., Wen S. R., Wang X. Y., J. Peking Univ. Heal. Sci., 18, 140-
147 (1986).

3) Wang J. M., Renshen Yanjiu, 13, 2-10 (2001).

4) Tashiro S., TDM Res., 5, 54 (1988).

5) Xie H. T., Wang G. J., lv H., Wang R., Sun J. G., Jiang X. L., Li H., Wang W., Huang C. R., Xu M. J., Eur. J. Drug Metab. Pharmacokinet., 30, 63-67 (2005).

6) Xie H. T., Wang G. J., Sun J. G., Tucker I., Zhao X. C., Xie Y. Y., Li H., Jiang X. L., Wang R., Xu M. J., Wang W., J. Chromatogr. B, 818, $167-173$ (2005).

7) Sun J. G., Wang G. J., Xie H. T., Li H., Pan G. Y., Tucker I., J. Pharm. Biomed. Anal., 38, 126-132 (2005).

8) Gong F., Liang Y. Z., Xie P. S., Chau F. T., J. Chromatogr. A, 1002 , $25-40(2003)$. 